\title{
EXTRAMAMMARY PAGET'S DISEASE VERSUS LICHEN SCLEROSUS
}

\author{
PAPPOVA, T. ${ }^{1}$, PEC, J. $^{1}$, KOZAROVA A. ${ }^{1}$, ADAMICOVA, K. ${ }^{2}$ \\ ${ }^{1}$ Comenius University Jessenius Faculty of Medicine and University Hospital, Department of Dermatovenerology, \\ Martin, Slovak Republic \\ ${ }^{2}$ Comenius University, Jessenius Faculty of Medicine and University Hospital, Department of Pathology, Martin, \\ Slovak Republic
}

\begin{abstract}
Burning, itching and dyspareunia are typical symptoms of many genital diseases. These subjective complaints can be misdiagnosed because of different clinical presentations. We present a case report of a postmenopausal woman treated for genital warts over a period of three years followed by the development of leukoplastic lesions in the whitish area clinical classified as Lichen sclerosus (LS). Histology of this lesion revealed carcinoma in situ. After radical surgical removal, vulvar Paget's disease was histologically verified. LS and extramammary Paget's disease (EMPD) belong to a group of uncommon dermatoses which mainly affect the skin of the genitals in postmenopausal women. Ulceration, erosions and leukoplastic lesions can signalize the development of squamous cell carcinoma in association of lichen sclerosus, on the other hand, they can be the sign of EMPD after a long period of time using different topical agents. The importance of reaching the correct diagnosis is essential and can influence current patient investigations and invasive or non-invasive treatment.
\end{abstract}

Key words: Lichen sclerosus, extramammary Paget’s disease, leukoplastic lesion

\section{INTRODUCTION}

Paget's disease was first described by Sir James Paget in 1874 as a rare intraepithelial neoplasm of the nipple associated with an underlying breast carcinoma mass (1). The following years, this carcinoma has also been reported in other parts of the skin and termed EMPD. This uncommon form of an intraepithelial neoplasm involves regions rich in apocrine glands, most commonly female genital organs, but also perianal, perineal, scrotal or penile regions. Less commonly the axilla, eyelids, external ear canal or umbilicus may be involved (2). EMPD represents only about $2 \%$ of all vulvar tumor diseases (3). We report a case of a postmenopausal woman with primary vulvar EMPD originally clinically diagnosed as squamous cell carcinoma arising from LS.

\section{CASE REPORT}

A 52-years-old woman patient admitted to our department suffered from anogenital warts and recurrent colpitis. The mentioned pathologic conditions had been treated by imiquimod and a wide variety of local antibacterial, antimycotic and corticosteroid agents for the last three years without results. Our physical examination revealed white, shiny, smooth patches with fissures at the right posterior commissure of the vulva and around the anus. In addition, leucoplastic lesions were present at the right posterior commissure of the vulva with a width of $1 \mathrm{~cm}$ and at the left part of the anal area with $2,5 \mathrm{~cm}$ in diameter (Fig. 1). Biopsies of these lesions were reported as high grade dyplastic changes with marks of „carcinoma in situ” with the presence of goblet cells (Fig.2). The presence of HPV infection was not detected by histological examination, neither by real-time PCR method. A partial vulvectomy and removal of the rest of the involved perianal region were performed to exclude squamous cell carcinoma. Hematoxylin eosin stained sections of vulva revealed squamous hyperplasia and large cells with clear cytoplasm and hyperchromatic nuclei located within the whole epidermis. These cells were interpreted as Paget cells. 
Paget cells infiltrated every layer of the epidermis and extended into adnexal structures (Fig. 3). Tumor infiltration into papillary dermis was detected (the depth was less than $1 \mathrm{~mm}$ ). Immunohistochemical studies demonstrated immunoreactivity of Paget cells to cytokeratin 7 (CK7), carcinoembryonic antigen (CEA) and gross cystic disease fluid protein-15 (GCDFP-15). The tumor cells were not immunoreactive for CK 20. The resection margins were free of neoplasia. Perianal tissue was with no evidence of tumor infiltration. A small motile resistance arising from the outer margin of the anal canal was seen during a subsequent visit for follow up (Fig. 4). Surgical removal of this lesion was performed and histological examination confirmed Paget's disease with the margins free of Paget cells. Colonoscopy and cystoscopy were performed in order to rule out adenocarcinoma. Control biopsies from the anal canal and rectal infundibulum did not reveal cancerous invasion. Similarly, neither CT scan of the abdomen and pelvis, performed to complete examinations, detected the presence of neoplasia.

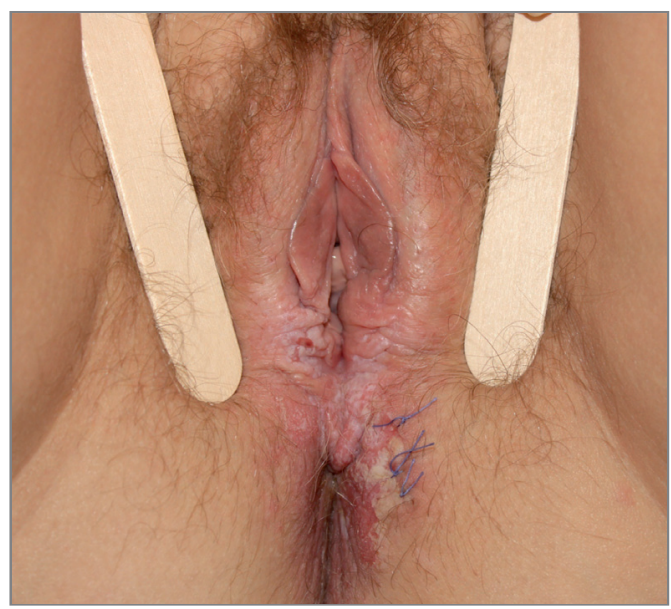

Fig. 1 Leucoplaplastic lesions at the right posterior commissure of the vulva and at the left part of the anal area in the whitish smooth area

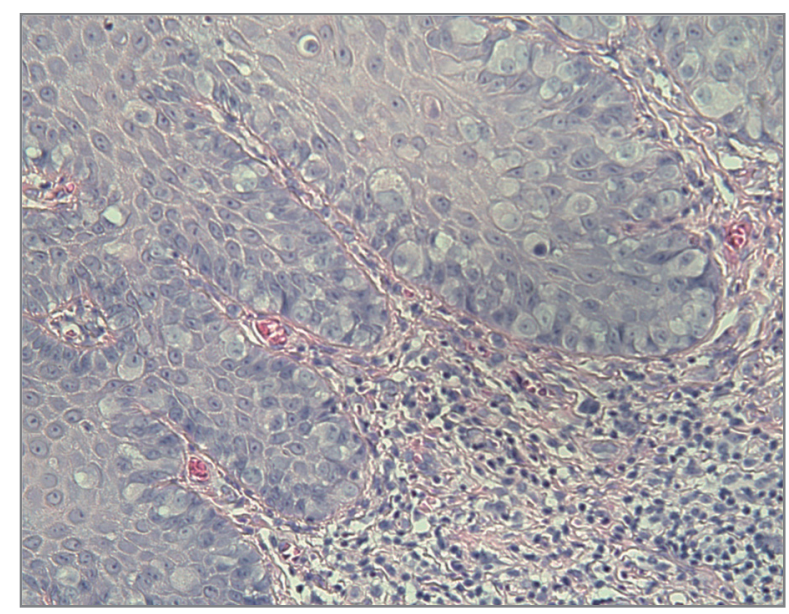

Fig. 2 High grade dyplastic changes with marks of „carcinoma in situ” with the presence of goblet cells (Hematoxylin eosin, magnifying 20x)

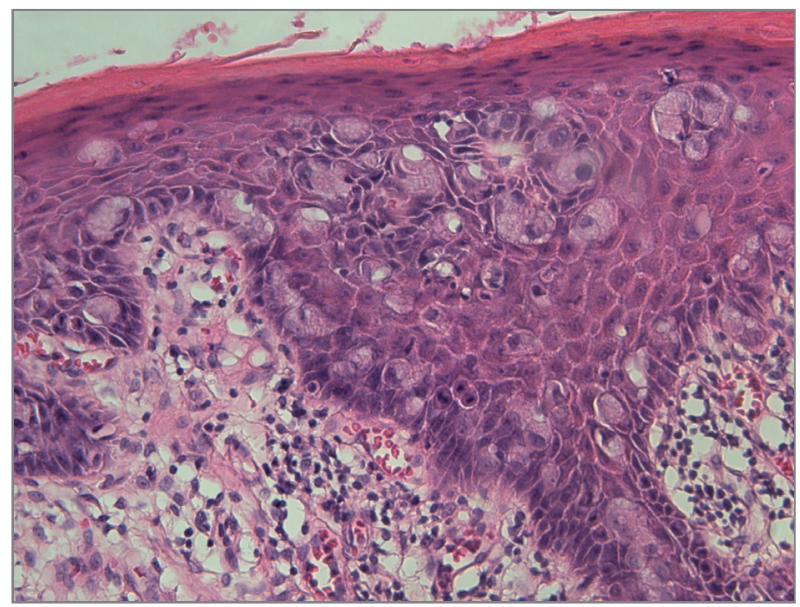

Fig. 3 Paget cells whitin the epidermis (Hematoxylin eosin, magnifying 20x)

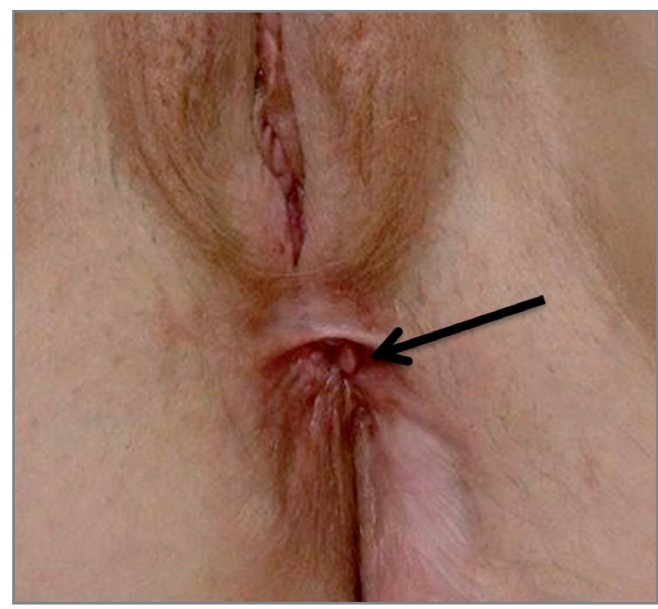

Fig. 4 Small motile resistance arising from the outer margin of the anal canal 


\section{DISCUSSION}

A lot of genital diseases are characterized by the similar symptomatology as itching, burning or dyspareunia. These troublesome symptoms are common features of LS and EMPD, two diseases which affect mainly postmenopausal women.

The characteristic lesions for LS are porcelain-white papules and plaques which become sclerotic and atrophic. Later stages can lead to complete loss of the labia minora, sealing of the clitoral hood, and burying of the clitoris. Since these lesions are fragile, erosions, fissures, purpura and ecchymoses are frequent $(4,5)$. However EMPD manifests as an erythematous, eczematous welldefined plaque, in some cases can have hyperpigmented, hypopigmented or depigmented macular appearance and so this disease can imitate LS. As well as LS also EMPD may present erosion and ulceration (6). Isolated depigmented changes of the skin are extremely rare and up to date, there are only 4 cases documented in the available literature (7-10). Furthermore, to our knowledge, there is only one case report similar to ours, where EMPD clinically imitates LS (11).

The intraepidermal origin of EMPD is poorly clarified. Paget cells may come either from adnexal structures, especially from apocrine glands or from multipotent stem cells located in the basal cell layer (12). According to the pathogenesis, the EMPD is divided into a primary and secondary form. The origin of primary EMPD, also termed cutaneous EMPD, is suggested to stem from epidermal basal cells. It can extend into adnexal structures and extraordinarily it causes dermal invasion. Sometimes EMPD can arise from underlying skin appendages such as apocrine glands and affects the overlying epidermis by epidermothropism (13). Secondary EMPD is a manifestation of noncutaneous internal malignancy, mainly anal, rectal or urothelial adenocarcinomas, which affects the skin either by epidermotropic metastases or by direct extension. A few cases of vulvar EMPD associated with distant internal tumors without a direct epithelial connection to the affected epidermis such as ovarian carcinoma, bile duct carcinoma, hepatocellular carcinoma, renal cell carcinoma or breast carcinoma, have been described in literature $(3,14)$.

Wilkinson and Brown proposed a classification of vulvar Paget's disease based on the etiology. They subdivided primary cutaneous vulvar Paget's disease to a) a primary intraepithelial neoplasm, b) an intraepithelial neoplasm with invasion, c) a manifestation of an underlying primary adenocarcinoma of a skin appendage or a subcutaneous vulvar gland. According to the origin also secondary vulvar Paget's disease was subdivided to a) secondary to anal or rectal adenocarcinoma, b) secondary to urothelial neoplasia, c) secondary to adenocarcinoma or related tumors of other sites (14).

Based on histological findings of presenting patient, all obtained evidence pointed to a primary form of EMPD. Paget cells were found in vulvar and perianal region. As it is well known, Paget cells can spread horizontally and vertically in macroscopically intact tissue, which can make difficult to determine the surgical margins (3), it is necessary to perform excisions with sufficient depth and width. For that reason, our patient submitted to a total excision of all visible lesions with at least $1 \mathrm{~cm}$ margin of excision and to the removal of all underlying skin appendages.

In our case, histological confirmation of dermal invasion may interfere with the determination of tumor origin. Thus, all needed examinations were performed to exclude secondary EMPD, leading to conclusion that there were no signs of rectal, anal and urogenital adenocarcinoma.

Medical care for the patient continues with regular follow up in dermatologic, gynaecologic and urologic clinics. Follow up is necessary because of the possibility of disease recurrence which is estimated to be $30-50 \%$ (15).

\section{CONCLUSION}

Genital dermatoses must be properly evaluated and must be taken as a part of differential diagnosis. Probatory biopsies are necessary, especially if fissures, plaques or ulceration are present. Histological findings can propose a new clinical view and so change the treatment management. 


\section{REFERENCES}

1. Paget, J. On disease of the mammary areola preceding cancer of the mammary gland. St. Bartholomew's Hospital Report 1874; 10: 87-89.

2. Zollo JD, Zeitouni NC. The Roswell Park Cancer Institute experience with extramammary Paget's disease. Brit J Dermatol 2000; 142 (1): 59-65.

3. Lloyd J, Flanagan A M . Extramammary Paget's disease with internal malignancies. J Clin Pathol 2000; 52 (10): 742-749.

4. Funaro D. Lichen sclerosus: a review and practical approach. Dermatologic Therapy 2004; 17 (1): 28-37.

5. Fistarol SK, Itin HP. Diagnosis and Treatement of Lichen Sclerosus. Am J Clin Dermatol 2013; 14 (1): 27-47.

6. Vanessa D., Andretta Tanaka et al. Mammary and Extramammary Paget's Disease: A Study of 14 Cases and the Associated Therapeutic Difficulties. Clinics (Sao Paulo) 2009; 64 (6): 599-606.

7. Chen YH, Wong TW, Lee JY. Depigmented genital extramammary Paget's disease: a possible histogenetic link to Toker's clear cells and clear cell papulosis. J Cutan Pathol 2001; 28 (2): 105-108.

8. Sawamura D, Yamamoto M, Fukushi G. A case of extramammary Paget's disease featuring depigmentation. Aomori Ken Byo Shi 1987; 32: 114.

9. Sawamura, D, Ishikawa, et al.: Depigmented macula as an initial manifestation of extramammary Paget's disease. J Dermatol 1996; 23(6): 429.

10. Kakinuma H, Iwasawa U et al. A case of extramammary Paget's disease with depigmented macules as the sole manifestation. Brit J Dermatol 1994; 130 (1): 102-105.

11. Bansal D, Bowman CA . Extramammary Paget's disease masquerading as lichen sclerosus. Int J STD \& AIDS 2004; 15(2): 141-142.

12. Regauer S. Extramammary Paget's disease-a proliferation of adnexal origin? Histopathology 2006; 48(6):723-9.

13. Nowak MA, Guerriere-Kovach P. Et al. Perianal Paget's disease: Distinguishing primary and secondary lesions using imunohistochemical studies including gross cystic disease fluid protein-15 and cytokeratin 20 exresion. Arch Pathol Lab Med. 1998; 122 (12): 1077-81.

14. Wilkinson EJ, Brown HM. Vulvar Paget Disease of Urothelial Origin: A Report of Three Cases and a Proposed Classification of Vulvar Paget Disease. Human Pathol 2002; 33 (5): 549-554.

15. De Magnis A, Checcucci V et al. Vulvar paget disease: a large single-centre experience on clinical presentation, surgical treatment, and long-term outcomes. J Lower Genit Tract Dis 2013; 17 (2): 104-1 10.

Received: October, 10,2016

Accepted: November, 16,2016 